\title{
Implementing a postdischarge methicillin-resistant Staphylococcus aureus decolonization protocol within a Veterans Affairs Health Care System facility
}

\author{
Matthew D. Hellman PharmD ${ }^{1}$, Kelly W. Davis PharmD², Brenda Hackworth BSN ${ }^{3}$ and Martin E. Evans MD ${ }^{4,5,6}$ (1) \\ ${ }^{1}$ Department of Pharmacy, Veterans Affairs Health Care System, Alexandria, Louisiana, ${ }^{2}$ Department of Pharmacy, Veterans Affairs Health Care System, \\ Lexington, Kentucky, ${ }^{3}$ Department of Cardiology, Veterans Affairs Health Care System, Lexington, Kentucky, ${ }^{4} \mathrm{VHA}$ National Infectious Diseases Service, Veterans \\ Healthcare Administration, Cincinnati, Ohio, ${ }^{5}$ Infectious Disease Service, Veterans Affairs Health Care System, Lexington, Kentucky and ${ }^{6}$ Division of Infectious \\ Diseases, Department of Internal Medicine, University of Kentucky School of Medicine, Lexington, Kentucky
}

To the Editor-Approximately $60 \%$ of methicillin-resistant Staphylococcus aureus (MRSA) infections occur after hospital discharge in Veterans Affairs (VA) patients who are colonized with the organism as inpatients. ${ }^{1}$ A recent multicenter, randomized, controlled trial (the CLEAR Trial) demonstrated a $44 \%$ reduction in such infections using a postdischarge decolonization regimen of chlorhexidine body rinse and mouthwash and nasal mupirocin. ${ }^{2}$ The CLEAR trial employed monthly phone calls, unit dose medications, and monetary incentives to improve protocol adherence. Unlike the CLEAR Trial, which was funded in part by the Agency for Healthcare Research and Quality, patient coaching, monetary incentives, and monthly calls to promote adherence with the decolonization regimen may be impractical in some VA medical centers due to fiscal and personnel constraints. However, we reasoned that if the CLEAR Trial decolonization protocol could be implemented within the Veterans Healthcare Administration (VHA), it may decrease postdischarge MRSA infections. We conducted a pilot study to test this hypothesis.

Patients from October 1, 2019, through April 1, 2020, were identified for enrollment into the Lexington VA study using a software program that mines VA Corporate Data Warehouse and identifies all patients with a history of MRSA colonization or infection within the past 12 months and who were admitted to the medical center within the past 24 hours. All admissions were additionally screened for eligibility using the VA Computerized Patient Record System. Patients were eligible for recruitment if they had the ability to bathe or shower (alone or with assistance) and were likely to be discharged home. Patients were excluded if they were under hospice care, allergic to the decolonization products, demented, admitted to the mental health unit, or were to be discharged to a long-term care facility.

Study participants were enrolled for a 1-year period consisting of 6 months of decolonization followed by 6 months of observation. The MRSA decolonization protocol was daily $4 \%$ chlorhexidine body rinse shower or bath, twice daily $0.12 \%$ chlorhexidine mouth rinse, and twice daily $2 \%$ mupirocin ointment applied to both nares. There was no cost to participants. Participants were instructed to perform decolonization for 5 days twice monthly for a period of 6 months after hospital discharge. A waterproof shower calendar

\footnotetext{
Author for correspondence: Martin E. Evans, E-mail: martin.evans@va.gov Cite this article: Hellman MD, et al. (2022). Implementing a postdischarge methicillinresistant Staphylococcus aureus decolonization protocol within a Veterans Affairs Health Care System facility. Infection Control \& Hospital Epidemiology, 43: 1276-1277, https:// doi.org/10.1017/ice.2021.225
}

was provided specifying which days to perform the protocol along with written and in-person education on the proper use of decolonization products. Study products were dispensed as 30-day supplies, and refills were tracked by the Lexington VA Pharmacy to evaluate protocol adherence. Full adherence was defined as 4-5 refills; partial adherence was defined as 2-3 refills; and nonadherence was defined as $0-1$ refills. Refilling even 1 of the 3 study products each month was considered a qualifying refill. This study was approved by the Lexington VA Institutional Review Board.

In total, 44 patients were enrolled in the trial among 301 screened for inclusion. Reasons for not being recruited included having a negative admission nasal swab $(n=64)$, declining to participate $(n=48)$, being missed by recruiters $(n=11)$, or other reasons $(n=75)$. The most common other reasons were being a nursing home resident, demented, a mental health patient, homeless, or in hospice. Moreover, $34 \%$ of patients discontinued the protocol because of death $(n=9)$, unexpected admission to a long-term care facility $(n=5)$, or at their request $(n=2)$. No patient was fully adherent with the decolonization protocol, $6(13.6 \%)$ were partially adherent, and $38(86.4 \%)$ were nonadherent.

Our study has several limitations. The most readily available and cost-effective product sizes were dispensed. As a result, the mupirocin ointment and chlorhexidine body wash may have lasted longer than 30 days and may have led us to underestimate adherence. On the other hand, the chlorhexidine oral rinse had a measurable volume that should have lasted only 30 days, yet this product was never refilled more than twice by any subject during the 6-month study period consistent with overall adherence being poor. Another limitation was the small study population with only $44(15 \%)$ of 301 screened admissions enrolled. The results may have been more robust with larger numbers. However, these numbers also reflect the difficulty of recruiting patients into this decolonization protocol and is consistent with a poststudy survey showing that many patients did not perceive their health as being better because of the decolonizing regimen (data not shown).

In this pilot study, $86 \%$ of patients at the Lexington VA did not adhere to the MRSA decolonization protocol used in the CLEAR Trial. Our trial may have been more effective if we could have provided the monthly adherence calls, convenient unit dose medications, and/or monetary incentives utilized in the CLEAR Trial. In the CLEAR Trial, most MRSA carriage reduction occurred in the first month of the decolonization protocol. ${ }^{3}$ A shorter decolonization course may be more acceptable to patients and still achieve the benefits sought. 


\section{Acknowledgments.}

Financial support. No financial support was provided relevant to this article.

Conflicts of interest. All authors report no conflicts of interest relevant to this article.

\section{References}

1. Nelson RN, Evans ME, Simbartl L, et al. Methicillin-resistant Staphylococcus aureus colonization and pre- and posthospital discharge infection risk. Clin Infect Dis 2019;68:545-553.
2. Huang SS, Singh R, McKinnell JA, et al. Decolonization to reduce postdischarge infection risk among MRSA carriers. N Engl J Med 2019;380: 638-650.

3. Huang SS, Singh RD, Eells SJ, et al. Impact of post-discharge chlorhexidine and mupirocin on MRSA carriage in a randomized trial. In: Program and abstracts of IDWeek 2013, October 2-6, 2013; San Francisco, CA. Abstract 1815.

\title{
bla $_{V I M}$ in wastewater drains: A hidden circulation of VIM-producing Enterobacterales in the hospital setting?
}

\author{
Carole Lemarié $\mathrm{MD}^{1}$, Estelle Marion $\mathrm{PhD}^{2}$, Rafael Mahieu MD MSc${ }^{2,3}$, Anne Donnars $\mathrm{MSc}^{1}$ and \\ Matthieu Eveillard PharmD, PhD ${ }^{1,2}$ (1) \\ ${ }^{1}$ Laboratoire de bactériologie, Département des agents infectieux, CHU Angers, 4 rue Larrey, F-49933, Angers, France, ${ }^{2}$ Centre de Recherche en cancérologie et \\ Immunologie Nantes-Angers Inserm, Université de Nantes, Université d'Angers, Angers, Nantes, France and ${ }^{3}$ Service des Maladies infectieuses et tropicales, CHU \\ Angers, France
}

To the Editor-Infections with carbapenemase-producing Enterobacteriaceae (CPE) are an increasing threat to public health. ${ }^{1}$ The risk of in-hospital mortality due to CPE bloodstream infection is considerably greater than that for carbapenemasesusceptible bloodstream infections. In France, VIM-producing Enterobacteriaceae represent $<5 \%$ of all CPE. ${ }^{2}$ In our teaching hospital in western France, only 3 patients with VIM-producing Enterobacter cloacae had been identified before January 2020 (November 2015, October 2016, and December 2018). These patients had been hospitalized in 3 different wards and had been fortuitously identified by rectal screening, with lengths of hospitalization preceding positive screening of 6, 34, and 204 days, which could suggest in-hospital acquisitions. We questioned whether these 3 cases were really isolated or if additional but undetected cases did or could occur. Concurrently, concerns are growing over the importance of the hospital water environment as a long-term reservoir of $\mathrm{CPE} .{ }^{3-5} \mathrm{We}$ investigated the potential role of wastewater drains in the hidden circulation of VIM-producing Enterobacteriaceae.

The study was performed in a 1,500-bed French teaching hospital. CPE carriage is systematically screened by rectal swabbing patients hospitalized in the intensive care units, at the time of admission, and once each week during hospitalization. In the other wards, CPE carriage is screened at the time of admission in patients who have been hospitalized in a foreign country within the preceding year and for contact cases. Wastewater drain sampling was performed in December 2019 in 4 intensive care units (ICUs),

Author for correspondence: Matthieu Eveillard, E-mail: MaEveillard@chu-angers.fr Cite this article: Lemarié $C$, et al. (2022). bla $a_{V I M}$ in wastewater drains: A hidden circulation of VIM-producing Enterobacterales in the hospital setting?. Infection Control \& Hospital Epidemiology, 43: 1277-1278, https://doi.org/10.1017/ice.2021.234
11 medical units and 3 surgical units. In the ICUs, all sink drains of patient rooms were sampled (1 sink drain in each room). Outside the ICUs, the 3 rooms in which VIM-positive patients had been hospitalized were sampled, and 30 other rooms were randomly chosen for sampling. In each of these rooms, the sink drain and the shower drain were sampled. Samples were performed by inserting eSwab sterile swabs (Copan Italia, Brescia, Italy) to a depth of $\sim 5 \mathrm{~cm}$ in each drain and rotating them to collect specimens from the inner wall of the drain for a minimum of 3 insertions. Specimens were stored at $4^{\circ} \mathrm{C}$ before culture, and an aliquot of the eSwab broth was immediately stored at $-80^{\circ} \mathrm{C}$ before molecular analysis. Swabs were plated onto selective agar plates (CHROMID Carba Smart, bioMérieux, Marcy l'Etoile, France). No enrichment in nutrient broth was performed before plating. Identification of suspicious colonies was performed by matrixassisted laser desorption/ionization-time of flight mass spectrometry using a VITEK MS mass spectrometer (bioMérieux). For suspicious colonies, carbapenemases were detected by immunochromatography (RESIST-4 O.K.N.V., Coris Bioconcept, Gembloux, Begium). A confirmation was planned, if appropriate, using the method of combined test (Rosco Diagnostica, Taastrup, Denmark). To increase the sensitivity of the screening, detection of carbapenemase genes was performed in the eSwab broth by qualitative real-time polymerase chain reaction (PCR) with a GeneExpert System (Xpert Carba-R; Cepheid, Sunnyvale, CA), which allowed us to identify $b l a_{\text {OXA-48, }}$, $b l a_{\mathrm{KPC}}, b l a_{\mathrm{IMP}}, b l a_{\mathrm{NDM}}$, and $b l a_{\mathrm{VIM}}$.

Overall, 102 wastewater drains (69 sink drains and 33 shower drains) were sampled from 36 rooms in ICUs and 33 rooms in the other wards. The results of cultures and PCR are presented in Table 1. We identified 29 carbapenemase genes in 26 rooms: 15 rooms in ICUs and 11 rooms in the other wards. Therefore, the 